\title{
Gerontology
}

\section{Comparison of Disease Clusters in Two Elderly Populations Hospitalized in 2008 and 2010}

\author{
A. Marengoni $^{a} \quad$ A. Nobili $^{b} \quad$ C. Pirali ${ }^{a} \quad$ M. Tettamanti ${ }^{b} \quad$ L. Pasina ${ }^{b} \quad$ F. Salerno ${ }^{c}$ \\ S. Corrao $^{d} \quad$ A. lorio $^{g}$ M. Marcucci ${ }^{g}$ C. Franchie P.M. Mannuccif ${ }^{f}$ on behalf of \\ REPOSI Investigators ${ }^{1}$
}

${ }^{a}$ Geriatric Unit, Spedali Civili, Department of Medical and Surgery Sciences, University of Brescia, ${ }^{b}$ Department of Neuroscience, Mario Negri Institute for Pharmacological Research, Milano, ' Medicina Interna, IRCCS Policlinico San Donato, Department of Medical and Surgery Sciences, University of Milano, ${ }^{\mathrm{d} D i p a r t i m e n t o ~ B i o m e d i c o ~ d i ~}$ Medicina Interna e Specialistica, University of Palermo, ' ${ }^{2}$ Laboratory for Quality Assessment of Geriatric Therapies and Services, Mario Negri Institute for Pharmacological Research, Milano, ${ }^{\mathrm{f} S c i e n t i f i c ~ D i r e c t i o n, ~ I R C C S ~ C a ̀ ~ G r a n d a ~}$ Maggiore Policlinico Hospital Foundation, Milano, Italy, and ${ }^{9}$ McMaster University, Hamilton, Canada

\section{Key Words}

Chronic diseases - Cluster analysis · Hospitalized elderly

\begin{abstract}
Background: As chronicity represents one of the major challenges in the healthcare of aging populations, the understanding of how chronic diseases distribute and co-occur in this part of the population is needed. Objectives: The aims of this study were to evaluate and compare patterns of diseases identified with cluster analysis in two samples of hospitalized elderly. Methods: Data were obtained from the multicenter 'Registry Politerapie SIMI (REPOSI)' that included people aged 65 or older hospitalized in internal medicine and geriatric wards in Italy during 2008 and 2010. The study sample from the first wave included 1,411 subjects enrolled in 38 hospitals wards, whereas the second wave included 1,380 subjects in 66 wards located in different regions of
\end{abstract}

Italy. To analyze patterns of multimorbidity, a cluster analysis was performed including the same diseases (19 chronic conditions with a prevalence $>5 \%$ ) collected at hospital discharge during the two waves of the registry. Results: Eight clusters of diseases were identified in the first wave of the REPOSI registry and six in the second wave. Several diseases were included in similar clusters in the two waves, such as malignancy and liver cirrhosis; anemia, gastric and intestinal diseases; diabetes and coronary heart disease; chronic obstructive pulmonary disease and prostate hypertrophy. Conclusion: These findings strengthened the idea of an association other than by chance of diseases in the elderly population.

Copyright $\odot 2013$ S. Karger AG, Basel

\footnotetext{
${ }^{1}$ REPOSI stands for REgistry of POlytherapies SIMI (Società Italiana di Medicina Interna). The investigators and co-authors of the REPOSI Study Group are listed in the Appendix.
}

\section{KARGER}

Fax +4161306 1234

E-Mail karger@karger.ch

www.karger.com
(C) 2013 S. Karger AG, Basel

$0304-324 \mathrm{X} / 13 / 0000-0000 \$ 38.00 / 0$

Accessible online at:

www.karger.com/ger
Alessandra Marengoni, MD, $\mathrm{PhD}$

Geriatric Unit, Spedali Civili, Department of Medical and Surgery Sciences

University of Brescia, Piazzale Spedali Civili 1

IT-25123 Brescia (Italy)

E-Mail marengon@med.unibs.it 


\section{Introduction}

As chronicity represents one of the major challenges in the healthcare of aging populations, the understanding of how chronic diseases distribute and co-occur in this part of the population is really valuable. In the last decade, a quantitative assessment of multimorbidity (e.g. evaluation of the coexistence of multiple chronic diseases in the same person, whichever they are) has been done because it is necessary and functional in order to quantify the dimension of the problem at a population level and to grab the attention of healthcare providers [1]. Nevertheless, the exclusive use of a quantitative approach to this phenomenon fails to catch different patterns of coexisting diseases, potentially leading to inadequate care management. Advancement in the field may be represented by the development of a 'qualitative analysis' of multimorbidity, in terms of how different diseases distribute and aggregate in the population, and a 'cluster medicine' approach, in terms of the identification of specific disease clusters, defined as the co-occurrence of two or more specific chronic diseases in the same persons [2]. Indeed, it has been shown that complex information, such as multimorbidity, can be better recognized as patterns [3]. Clusters can be identified using different statistical tools, such as the multimorbidity coefficient, which is the ratio of the observed co-prevalence to the expected one (prevalence of the diseases if they are completely independent) of a set of diseases. This coefficient tells us the degree to which the co-morbid diseases exceed the chance level. However, in order to have a complete picture of how diseases distribute and co-occur in a population, there is a more informative method that can be used, called cluster analysis; clustering is simply the grouping of similar objects by using algorithms and different measures of similarity in order to group variables with the highest association.

The aim of this study was to evaluate and compare patterns of diseases identified with cluster analysis in two populations of hospitalized elderly. Data were obtained from the 'Registry Politerapie SIMI (REPOSI)' collected during 2008 and 2010 in internal medicine and geriatric wards in Italy.

\section{Methods}

\section{Data Collection}

The REPOSI (Registro Politerapie SIMI) is a collaborative effort between the Italian Society of Internal Medicine (SIMI) and the Mario Negri Institute of Pharmacological Research (Milan).
The REPOSI was designed with the purpose of creating a network of internal medicine and geriatric wards in order to evaluate patients affected by multiple diseases and prescribed with polytherapy. Participation in the network was on a voluntary basis, but in the choice of the participating centers, attention was given to their homogeneous composition in terms of geographic distribution, size, and unselected admissions from the territory or the emergency room. The specific aims of the REPOSI study were: to describe the prevalence of co-occurring multiple diseases and treatments in hospitalized elderly patients, to correlate clinical characteristics of the patients with type and number of diseases and treatments, and to evaluate the main clinical outcomes at hospital discharge. The original study design included two phases: phase one was designed to create the network of internal medicine and geriatric wards, and phase two was intended to activate a registry of patients included in the study. All of the patients admitted to the wards participating in the study were recruited consecutively if they were 65 years old or older. Participation in the study was voluntary and an informed consent was signed by all the patients [4].

The first wave of the REPOSI register was held between January and December 2008 in 38 hospitals located in different regions of Italy. A sample of at least 40 patients consecutively admitted to each participating center during a period of 4 weeks, 3 months apart each from the other (one in February, one in June, one in September, and one in December, 2008) was included in the study. A standardized web-based case report form was filled in by the attending physicians, including socio-demographic factors, clinical parameters, diagnoses and treatments at both hospital admission and discharge, clinical events during hospitalization, and outcome. The initial study sample included 1,411 subjects. Of these, 79 (5.6\%) were excluded due to missing or incomplete data, 25 had missing data on hospital outcome, and 54 on socio-demographic and clinical characteristics due to errors in data input and recording; 66 patients died during hospitalization, and 111 were transferred to other hospital units. Hence, 1,155 individuals from the first wave were available for the present analyses.

The second wave was conducted between January and December 2010 in 66 hospitals wards located in different regions of Italy. A sample of at least 20 patients consecutively admitted to each participating hospital during a period of 4 weeks, 3 months apart each from the other (one in February, one in June, one in September, and one in December 2010) was enrolled in the study. The initial study sample included 1,380 subjects. Of these, $120(8.6 \%)$ were excluded because they were transferred to other hospital wards and 50 (3.6\%) died during hospitalization; 37 had missing information. Hence, 1,173 individuals from the second wave were available for the analyses. All the data recorded in the net were collected and checked by a central monitor institution (the Mario Negri Institute for Pharmacological Research, Milan). The study was approved by the Ethical Committee of the IRCCS Cà Granda Maggiore Policlinico Hospital Foundation, Milan.

\section{Assessment of Diseases}

Diseases examined in this study were collected at hospital discharge after clinical examination, medical history, and laboratory and instrumental data were compiled and performed by the attending physicians. Diagnoses were made using standardized cri- 
teria. The International Classification of Diseases, Ninth Revision (ICD-9) [5] was used for classifying all the diseases. The following ICD-9 codes were employed (corresponding diseases are listed in alphabetical order): 280-285 (anemia), 300 (anxiety), 715 (arthritis), 427 (atrial fibrillation, AF), 430-438 (cerebrovascular diseases, CVD), 410-414 (coronary heart disease, CHD), 490-496 (chronic obstructive pulmonary disease, COPD), 585 (chronic renal failure, CRF), 290 and 331 (dementia), 250 (diabetes mellitus), 272 (dyslipidemia), 530-536 (gastric diseases), 428 (heart failure, HF), 401-405 (hypertension), 560-569 (intestinal diseases), 571 (liver cirrhosis), 140-165, 170-175, and 179-208 (malignancy), 600 (prostate hypertrophy), and 240-246 (thyroid diseases). Only diseases with a prevalence of at least $5 \%(n=19)$ were taken into account in this study.

\section{Statistical Analysis}

In order to analyze different patterns of multimorbidity, without any a priori hypothesis, a cluster analysis [6] was performed including the same diseases (chronic diseases with a prevalence at discharge $>5 \%$ ) during the two waves of the registry. With cluster analysis is possible to go beyond the simple pairs of diseases and considering how diseases tend to occur in conjunction with each other. The aim is to obtain a complete picture of the distribution of diseases in the population and identify where a specific disease appears in the patterns. It is an exploratory data analysis that gives insights into the structure of a dataset and may lead to hypotheses for further investigations. It is best seen as hypothesis-generating rather than -solving. Cluster analysis is a multivariate statistical technique; clustering is the grouping of similar objects by using algorithms in order to minimize the 'logical distance' inside each group and maximize it between groups. The logical distance is calculated according to measure of similarity/dissimilarity amongst variables. For the purpose of this study an agglomerative hierarchical cluster analysis was performed. A correlation matrix was computed among all the diseases using the Yule Q measure of similarity (a similarity measure for binary data that weighs up the number of positive matches and the number of negative matches) and average linkage as algorithm. The dendrograms resulting from the two cluster analyses were compared. The distribution of diseases seen in different clusters should be significantly different from random distribution.

\section{Results}

\section{REPOSI, First Wave}

Of the 1,155 patients that were included in the analyses, $53.6 \%$ were females. The median age of the patients was 79 years (65-101). The main characteristics of the patients, including reasons for hospital admission, are described in table 1. The most frequent diagnoses at hospital admission were: hypertension (60.6\%), diabetes mellitus (27.0\%), CVD (26.5\%), CHD (25.5\%), AF (25.0\%), and COPD (21.7\%) (table 2).

The aggregation of diseases was tested by using cluster analysis, and eight clusters were identified. Five clusters
Table 1. Main characteristics of participants at the first (2008) and second (2010) wave of REPOSI

\begin{tabular}{lll}
\hline & $\begin{array}{l}\text { First wave } \\
2008\end{array}$ & $\begin{array}{l}\text { Second wave } \\
2010\end{array}$ \\
\hline Number & 1,155 & 1,173 \\
Age, years, median & $79(65-101)$ & $79(65-100)$ \\
Female, \% & 53.6 & 48.2 \\
Education, years, mean (SD) & $6.4(2.3)$ & $7.1(4.1)$ \\
Most frequent reasons for hospital admission & \\
Dyspnea, \% & 6.1 & 8.5 \\
Pain, \% & 6.1 & 7.7 \\
Anemia, \% & 6.1 & 6.0 \\
Heart failure, \% & 6.0 & 7.3 \\
Syncope, \% & 3.5 & 4.5 \\
Fever, \% & 3.2 & 4.9 \\
\hline
\end{tabular}

Table 2. Prevalence (per 100) of diseases in the first and second waves of the REPOSI

\begin{tabular}{llcc}
\hline Diseases & First wave & $\begin{array}{l}\text { Second wave } \\
2010\end{array}$ \\
& 2008 & $\mathrm{p}$ & \\
\hline Hypertension & 60.6 & 78.1 & $<0.001$ \\
Diabetes mellitus & 27.0 & 27.2 & 0.921 \\
Cerebrovascular disease & 26.5 & 21.6 & $<0.01$ \\
Coronary heart disease & 25.5 & 24.1 & 0.458 \\
Atrial fibrillation & 25.0 & 24.7 & 0.720 \\
Chronic obstructive & & & \\
$\quad$ pulmonary disease & 21.7 & 25.2 & 0.05 \\
Anemia & 20.0 & 12.9 & $<0.001$ \\
Malignancy & 19.7 & 17.3 & 0.160 \\
Heart failure & 18.6 & 19.1 & 0.766 \\
Gastric diseases & 17.1 & 24.9 & $<0.001$ \\
Chronic renal failure & 14.5 & 17.1 & 0.06 \\
Dyslipidemia & 13.8 & 9.5 & $<0.001$ \\
Gut diseases & 11.1 & 13.9 & $<0.05$ \\
Thyroid diseases & 10.8 & 11.1 & 0.841 \\
Liver cirrhosis & 10.6 & 10.9 & 0.890 \\
Dementia & 9.1 & 7.5 & 0.190 \\
Anxiety & 8.4 & 9.4 & 0.451 \\
Prostate hypertrophy & 8.2 & 12.1 & $<0.01$ \\
Arthritis & 7.5 & 9.9 & $<0.05$ \\
& & &
\end{tabular}

included two diseases: one liver cirrhosis and malignancy, one COPD and prostate hypertrophy, one diabetes and $\mathrm{CHD}$, one dementia and arthritis, and one thyroid dysfunction and anxiety. Three clusters included three conditions: one anemia, gastric diseases and intestinal diseases, one HF, AF and CRF, and one hypertension, dyslipidemia and CVD (fig. 1). 
Fig. 1. Dendogram resulting from cluster analysis testing the distribution and aggregation of diseases in the REPOSI first wave (2008)

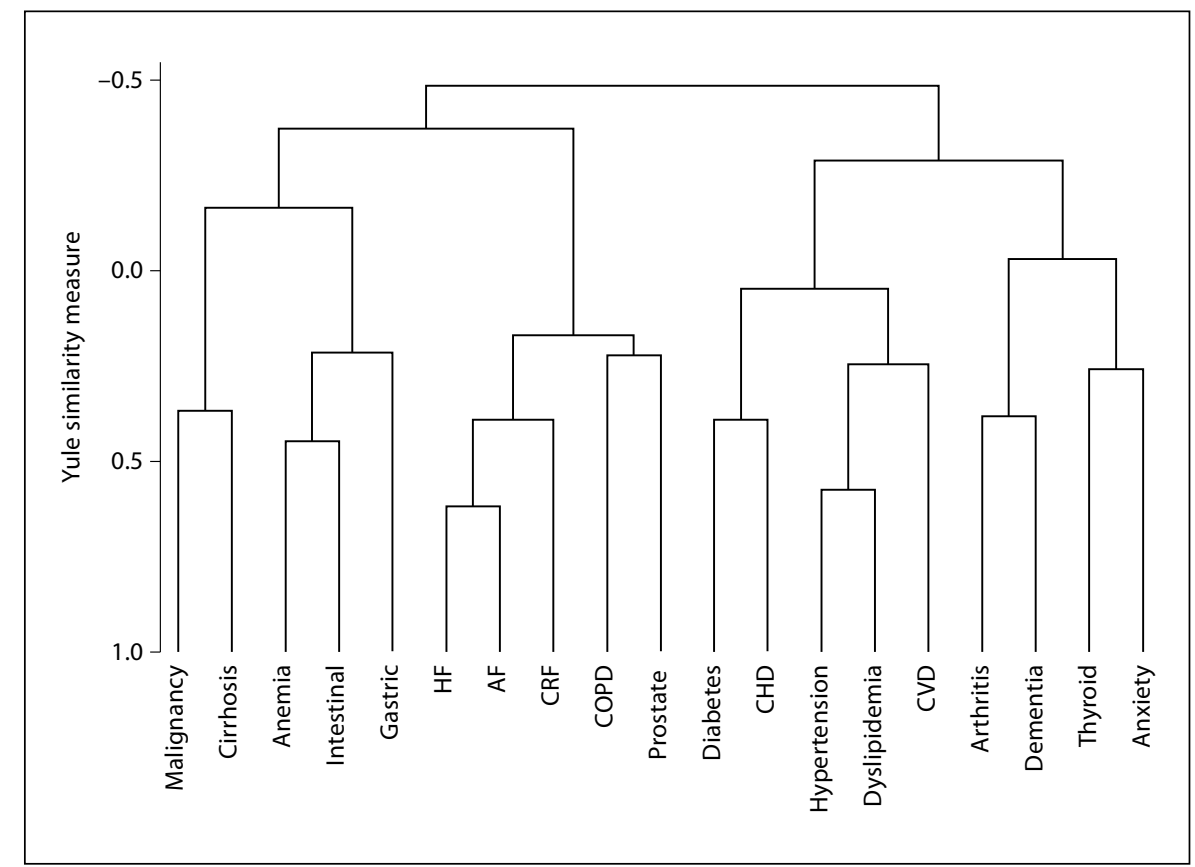

\section{REPOSI, Second Wave}

Of the 1,173 patients that were included in the analyses, $48.2 \%$ were females. The median age of the patients was 79 years (65-100). The main characteristics of the patients are described in table 1 . The most frequent diagnoses at hospital admission were: hypertension (78.1\%), diabetes mellitus (27.2\%), CHD (24.1\%), AF (24.7\%), COPD (25.2\%), and CVD (21.6\%) (table 2). In the second wave, the prevalence of hypertension, gastric and gut diseases, prostate hypertrophy and arthritis was significantly higher than the one found in the first wave; on the contrary, the prevalence of CVD, anemia and dyslipidemia was significantly lower (table 2).

Six clusters were identified using cluster analysis. One included only two diseases, liver cirrhosis and malignancy; three clusters included three diseases: one diabetes, CHD and dyslipidemia, one hypertension, thyroid dysfunction and AF, and one CVD, dementia and arthritis. Two clusters included four diseases: one anemia, gastric diseases, gut diseases and anxiety and one HF, CRF, COPD and prostate hypertrophy (fig. 2).

Several diseases were included in similar clusters in the two phases, such as malignancy and liver cirrhosis; anemia, gastric and intestinal diseases; diabetes and CHD; COPD and prostate hypertrophy. The main differences were that in the second wave, CVD aggregated with dementia instead of hypertension and dyslipidemia, and HF and CRF with COPD instead of AF; on the contrary, dyslipidemia aggregated with diabetes and $\mathrm{CHD}$, and AF with thyroid dysfunction and hypertension. An example of comparison between clusters identified in the REPOSI first and second wave is shown in figure 3 .

\section{Discussion}

Despite the increasing prevalence of chronic conditions with age, knowledge about how diseases distribute or co-occur in the same individual is limited [7]. Our knowledge is incomplete because few studies have attempted to describe the overall pattern of diseases and because alternative methods have been used making findings from different studies difficult to match up.

In the present study, a cluster approach was employed in order to evaluate and compare the distribution of diseases in two samples of hospitalized elderly across Italy in different time periods.

Eight clusters of diseases were identified in the first wave and six in the second wave. Several diseases were included in similar clusters in the two phases, such as malignancy and liver cirrhosis; anemia, gastric and intestinal diseases; diabetes and CHD; COPD and prostate hypertrophy. The main differences were that in the second wave, CVD aggregated with dementia instead of hypertension and dyslipidemia, and HF and CRF with COPD 
Fig. 2. Dendogram resulting from cluster analysis testing the distribution and aggregation of diseases in the REPOSI second wave (2010).

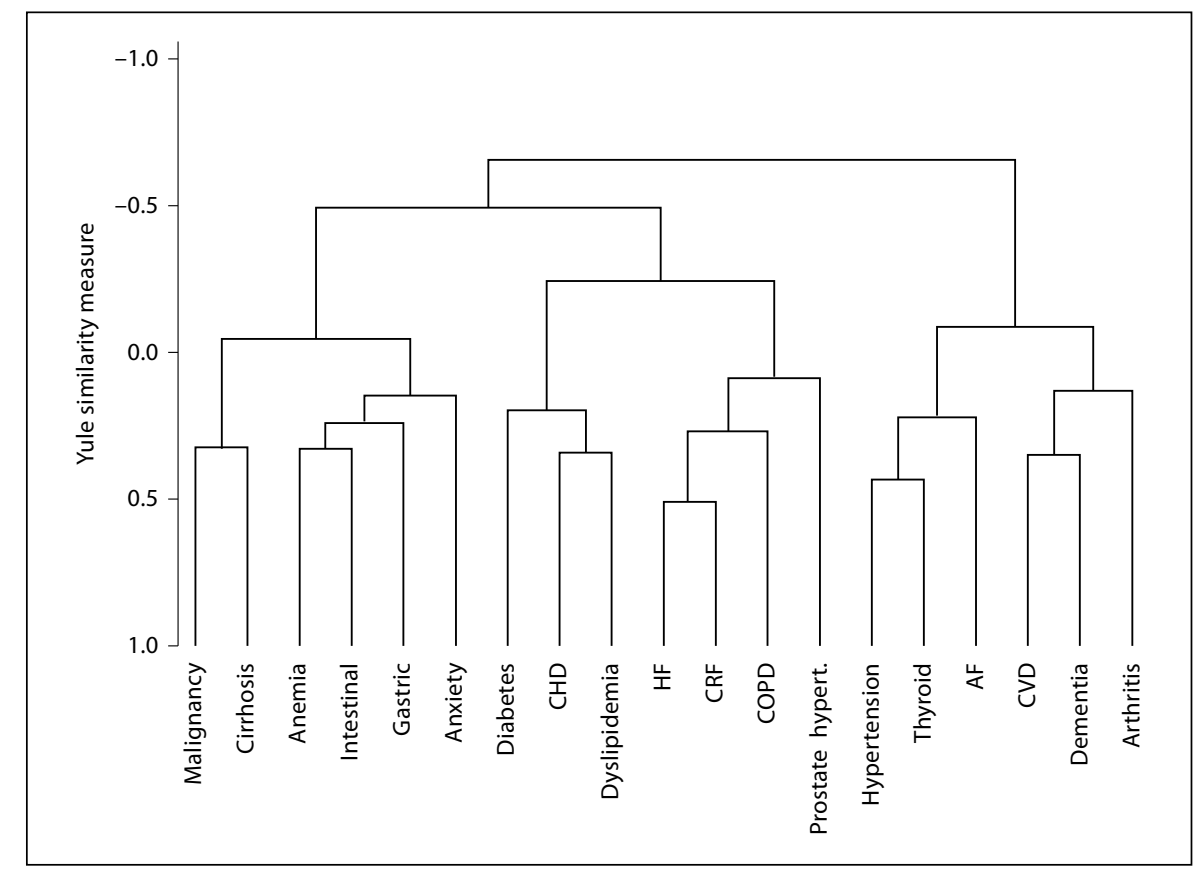

instead of AF; on the other hand, dyslipidemia aggregated with diabetes and $\mathrm{CHD}$, and $\mathrm{AF}$ with thyroid dysfunction and hypertension.

One possible explanation of the differences amongst clusters found in wave 1 and 2 probably relies in the different number and types of participating hospital units between the two phases of the study. This implies not only a difference in the prevalence of some chronic diseases, but also in admission policies that may vary from hospital to hospital. Besides, the meaningfulness of the distribution of diseases in patterns was maintained in both waves. For example, in the second wave, CVD aggregated with dementia and it is well known that vascular cerebral pathology can lead to cognitive impairment, especially in elderly people; dyslipidemia aggregated with CVD in the first and with CHD in the second wave being a risk factor for both the above vascular diseases.

Other studies have used the clustering approach in order to identify groups of associated diseases, in American [6], Swedish [8], and German [9, 10] adult and elderly people, but since now, research on diseases clusters has been mostly explanatory of the distribution and co-occurrence of diseases in a specific population. However, several potential uses of a cluster medicine approach deserve to be highlighted:

(1) New research hypotheses on possible shared pathological pathways for clusters of specific diseases can be developed. Differences in disease patterns may indicate

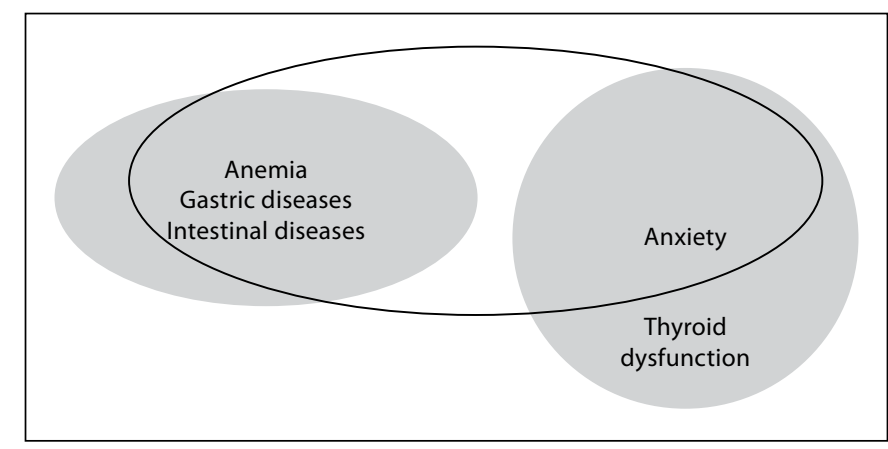

Fig. 3. Example of comparison of clusters of diseases identified in the REPOSI first and second waves. Shaded areas indicate clusters identified in the first wave (2008) and black ellipse indicates clusters identified in the second wave (2010) of the registry. In the first wave, anemia, gastric and intestinal diseases were included in one cluster and anxiety and thyroid dysfunction in a different cluster; in the second wave, anxiety were included in the same cluster as anemia, gastric and intestinal diseases.

differences in the biological process, environment or healthcare quality [11]. Nakauchi et al. [12] studied the relationship between chronic glomerulonephritis and infections and identified different clusters of immune responsiveness probably associated with different risks of graft rejection. Our findings showed an association between COPD and prostate hypertrophy in both waves. Despite this result they may depend on the higher preva- 
lence of COPD in elderly men and the hypothesis of a genetic common pathway, already demonstrated for COPD and prostate cancer [13], might be deepened also for COPD and prostate hypertrophy.

(2) Prevention, especially secondary prevention, can be implemented. In our study, we found patterns of diseases, some of which could be better targeted for secondary prevention, such as hypertension, dyslipidemia and CVD.

(3) Groups of people at high risk of adverse outcomes (e.g. disability or polypharmacy) can be identified. Previous findings from the REPOSI registry showed that specific clusters of diseases had a different impact on mortality and adverse clinical events during hospitalization [14]. Patients suffering from concurrent anemia and CRF had an additive risk of in-hospital death, whereas patients affected by HF and CRF had an additive risk of adverse events compared to their pairs affected by only one of the above diseases [14].

(4) The prevalence of use of potentially inappropriate medication or adverse drug reactions could be higher in different clusters. In a previous analysis of the REPOSI data, we found different degrees of association of specific clusters with prescription of polypharmacy at hospital discharge [15]. Besides, the mean number of prescribed drugs at hospital discharge ranged from 5.7 to 8.7 according to different disease clusters [15].

(5) Financial resources could be better distributed, in fact costs for specific clusters of diseases may be additive or multiplicative. Cluster analysis methods can be leveraged to develop targeted care management interventions designed to improve health outcomes. Newcomer et al. [16] used cluster analysis for identifying subpopulations of complex patients who may benefit from targeted care strategies.

(6) Ideally, once the triggering event (i.e. the onset of a specific disease) that promotes the clustering with other diseases has been identified, trials may be designed in order to change the chain of events. Moreover, clinical trials could be carried out in groups of elderly affected by specific clusters of diseases, e.g. the most easy/difficult to treat, the most expensive to treat or the ones with the best cost-effectiveness ratio [17].

(7) Finally, the severity of a disease can be approximated by its connections with other diseases for patients with the same number of diagnoses.

Major strengths of the REPOSI registry are the multicenter design that involved 38 and 66 internal medicine and geriatric wards throughout Italy respectively during 2008 and 2010, resulting in a sample representa- tive of the hospitalized elderly population in the country, and the inclusion of the patients during a period of 4 weeks (one per season) in order to balance the effect of seasons on acute diseases leading to hospitalization. However, a few limitations need to be mentioned. First, problems can arise by using hospital data for research purposes, because hospital records are not designed for research purposes but rather for patient care and their diagnostic quality may vary depending on different hospitals, physicians and clinical units. Second, hospital admissions are often selective on the basis of ward characteristics, severity of disease, associated medical conditions and admission policies that may vary from hospital to hospital. This could explain why the prevalence of some diseases was significantly different between waves 1 and 2, as in wave 2 the number of participating centers almost doubled. Finally, applying the cluster approach to the geriatric population has some limitation itself, such as the large number of participants required and, in this study, the cross-sectional design that does not allow conclusions regarding causal relationships among disease clusters. Moreover, unlike many other statistical procedures, cluster analysis methods are mostly used when researchers do not have any a priori hypothesis, but are still in the exploratory phase of research. In a sense, cluster analysis finds 'the most significant solution' possible. Therefore, statistical significance testing is not appropriate. Instead 'external stability' can be measured when small changes in the input data (such as little difference in prevalence of diseases) lead to only small changes in the classification achieved. The neighborhood of clusters, together with the vertical distances of the ramifications, is a measure of their similarities [3].

In conclusion, eight clusters of diseases were identified in the first wave of the REPOSI registry and six in the second wave. Several diseases were included in similar clusters in the two phases, such as malignancy and liver cirrhosis; anemia, gastric and intestinal diseases; diabetes and CHD; COPD and prostate hypertrophy. These findings strengthened the idea of an association other than by chance of diseases in the elderly population.

\section{Disclosure Statement}

The authors have no conflicts of interest to disclose.
Marengoni et al. 


\section{Appendix}

Investigators and co-authors of the REPOSI (REgistro POliterapie SIMI, Società Italiana di Medicina Interna) Study Group are as follows:

Steering Committee: Pier Mannuccio Mannucci (Chair, Fondazione IRCCS Cà Granda Ospedale Maggiore Policlinico, Milano), Alessandro Nobili (co-chair, Istituto di Ricerche Farmacologiche 'Mario Negri', Milano), Mauro Tettamanti, Luca Pasina, Carlotta Franchi (Istituto di Ricerche Farmacologiche 'Mario Negri', Milano), Francesco Salerno (IRCCS Policlinico San Donato Milanese, Milano), Salvatore Corrao (ARNAS Civico, Di Cristina, Benfratelli, Palermo), Alessandra Marengoni (Spedali Civili di Brescia, Brescia), Alfonso Iorio (McMaster University, Hamilton, Ont., Canada), Maura Marcucci (Azienda Ospedaliera Santa Maria della Misericordia, Perugia).

Clinical Data Monitoring and Revision: Valentina Spirito, Damia Noce, Jacopo Bonazzi, Rossana Lombardo, Eleonora Sparacio, Stefania Alborghetti (Istituto di Ricerche Farmacologiche 'Mario Negri', Milano).

Database Management and Statistics: Mauro Tettamanti, Luigi De Vittorio, Codjo Djignefa Djade (Istituto di Ricerche Farmacologiche 'Mario Negri', Milano).

Investigators: Giuseppe Paolisso, Maria Rosaria Rizzo, Maria Teresa Laieta (Azienda Ospedaliera Universitaria della Seconda Università degli Studi di Napoli, Napoli, VI Divisione di Medicina Interna e Malattie Nutrizionali dell'Invecchiamento); Torella Roberto, Marcello Persico, Teresa Salvatore, Ferdinando Carlo Sasso (Azienda Ospedaliera Universitaria della Seconda Università degli Studi di Napoli, Napoli, Medicina Interna e Malattie EpatoBilio Metaboliche Avanzate); Riccardo Utili, Emanuele Durante Mangoni, Daniela Pinto (Azienda Ospedaliera Universitaria della Seconda Università degli Studi di Napoli, Napoli, Medicina Infettivologica e dei trapianti); Luigi Fenoglio, Chiara Brignone, Christian Bracco (Azienda Sanitaria Ospedaliera Santa Croce e Carle di Cuneo, Cuneo, Medicina Interna); Laura Gasbarrone, Patrizia Porcari, Giseppe Famularo, Maria Rosaria Sajeva (Azienda Ospedaliera Ospedale San Camillo, Roma, Medicina Interna 1); Giorgio Maniscalco, Massimo Gunelli, Daniela Tirotta (Azienda Ospedaliera Ospedale San Salvatore, Pesaro, Soc Medicina Interna); Giuseppe Realdi, Aldo Baritussio, Francesco Frassoni (Azienda Ospedaliera Università di Padova, Padova, Clinica Medica I); Roberto Delsignore, Maria Cristina Baroni, Marianna Zardo (Azienda Ospedaliera Università di Parma, Parma, Clinica e Terapia Medica Renato Fellin), Stefano Volpato, Sioulis Fotini (Azienda Ospedaliera Universitaria Ospedale Sant'Anna, Ferrara, Unità Operativa di Medicina Interna Gerontologia e Geriatria); Roberto Manfredini, Carlo Longhini, Christian Molino, Elena Incasa (Azienda Ospedaliera Università Ospedale Sant'Anna, Ferrara, Unità Operativa Clinica Medica); Gianfranco Guarnieri, Michela Zanetti, Annalisa Spalluti (Azienda Ospedaliera Universitaria Ospedali Riuniti di Trieste, Trieste, Clinica Medica Generale e Terapia Medica); Giovanbattista Rini, Pasquale Mansueto, Ilenia Pepe (Azienda Ospedaliera Universitaria Policlinico P. Giaccone di Palermo, Palermo, Medicina Interna e Malattie Metaboliche); Giuseppe Licata, Luigi Calvo, Maria Valenti, Antonino Tuttolomondo, Riccardo Di Sciacca (Azienda Ospedaliera Universitaria Policlinico P. Giaccone di Palermo, Palermo, Medicina Interna e Cardioangiologia); Salvatore Antonaci, Francesco Vella, Alessandro Marseglia (Azienda Ospedaliero-Universitaria Consorziale
Policlinico di Bari, Bari, Clinica Medica II Frugoni); Vincenzo Centonze, Maria Ester Modeo (Azienda Ospedaliero-Universitaria Consorziale Policlinico di Bari, Bari, Medicina Interna Ospedale 'Pende-Ferrannini'); Giuseppe Palasciano, Stefania Pugliese, Caterina Capobianco (Azienda Ospedaliero-Universitaria Consorziale Policlinico di Bari, Bari, Medicina Interna Murri); Giovanni Murialdo, Marta Bovio (IRCS Azienda Ospedaliera Universitaria San Martino-IST di Genova, Genova, Clinica di Medicina Interna 2); Franco Laghi Pasini, Pier Leopoldo Capecchi, Maurizio Bicchi (Azienda Ospedaliera Universitaria Senese, Siena, Unità Operativa Complessa Medicina 2); Ranuccio Nuti, Roberto Valenti, Cosimo Capodarca (Azienda Ospedaliera Università Senese, Siena, Medicina Interna I); Alberto Auteri, Anna Laura Pasqui, Luca Puccetti (Azienda Ospedaliera Universitaria Senese, Siena, Medicina 3); Oliviero Olivieri, Anna Maria Stanzial (Azienda Ospedaliera Universitaria Integrata di Verona, Verona, Unità Operativa di Medicina Interna B); Giancarlo Agnelli, Alfonso Iorio, Maura Marcucci, Ana Macura (Azienda Ospedaliera Santa Maria della Misericordia, Perugia, Medicina Interna e Cardiovascolare); Elmo Mannarino, Graziana Lupattelli, Pamela Rondelli (Azienda Ospedaliera Santa Maria della Misericordia, Perugia, Medicina Interna, Angiologia, Malattie da Arteriosclerosi); Maria Grazia Serra (Azienda Ospedaliera 'Cardinale Panico' di Tricase, Lecce, Unità Operativa Complessa Medicina); Giuseppe Musca, Olga Cuccurullo (Azienda Sanitaria Provinciale di Cosenza Presidio Ospedaliero di Cetraro, Cosenza, Unità Operativa Complessa Medicina Interna); Maria Domenica Cappellini, Giovanna Fabio, Irene Motta, Flavio Cantoni (Fondazione IRCCS Cà Granda Ospedale Maggiore Policlinico, Milano, Unità Operativa Medicina Interna IA); Silvia Fargion, Paola Bonara, Mara Bulgheroni (Fondazione IRCCS Cà Granda Ospedale Maggiore Policlinico, Milano, Medicina Interna 1B); Fabio Magrini, Ferdinando Massari, Tatiana Tonella (Fondazione IRCCS Cà Granda Ospedale Maggiore Policlinico, Milano, Unità Operativa Medicina Cardiovascolare); Flora Peyvandi, Alberto Tedeschi, Raffaella Rossio (Fondazione IRCCS Cà Granda Ospedale Maggiore Policlinico, Milano, Medicina Interna 2); Guido Moreo, Barbara Ferrari, Luisa Roncari (Fondazione IRCCS Cà Granda Ospedale Maggiore Policlinico, Milano, Medicina Interna 3); Valter Monzani, Valeria Savojardo, Christian Folli (Fondazione IRCCS Cà Granda Ospedale Maggiore Policlinico, Milano, Medicina d'Urgenza); Daniela Mari, Paolo Dionigi Rossi, Eleonora Ziglioli, Carlo Vergani (Fondazione IRCCS Cà Granda Ospedale Maggiore Policlinico, Milano, Geriatria); Maria Sole Lilleri (Fondazione IRCCS Cà Granda Ospedale Maggiore Policlinico, Milano, Medicina Generale ad Indirizzo Geriatrico); Mauro Podda, Carlo Selmi, Francesca Meda (IRCCS Istituto Clinico Humanitas, Milano, Clinica Medica); Francesco Salerno, Massimo Cazzaniga, Valentina Monti (IRCCS Policlinico San Donato Milanese, Milano, Medicina Interna I); Carlo L. Balduini, Giampiera Bertolino, Luca Dezzani, Piergiorgio Cavallo (IRCCS Policlinico San Matteo di Pavia, Pavia, Clinica Medica III); Gino Roberto Corazza, Emanuela Miceli (IRCCS Policlinico San Matteo di Pavia, Pavia, Clinica Medica I, Reparto 11); Maria Beatrice Secchi, Sheng Chin Wu (Ospedale Bassini di Cinisello Balsamo, Milano, Divisione Medicina); Luigi Anastasio, Lucia Sofia, Maria Carbone, Leonardo Bertucci (Ospedale Civile Jazzolino di Vibo Valentia, Vibo Valentia, Medicina Generale); Giancarlo Traisci, Lucrezia De Feudis (Ospedale Civile Santo Spirito di Pescara, Pescara, Medicina Interna); Elisabetta Bergami, Emanuela Rizzioli (Ospedale del Del- 
ta, Lagosanto, Ferrara, Medicina Interna); Carlo Cagnoni, Luca Bertone, Antonio Manucra (Ospedale di Bobbio, Piacenza, Unità Operativa Medicina e Primo Soccorso); Esio Ronchi, Alberto Buratti, Tiziana Tognin, Daniele Bertolini, Nicola Lucio Liberato (Ospedale di Casorate Primo Carlo Mira, Pavia, Unità Operativa Medicina Generale); Giordano Bernasconi, Barbara Nardo (Ospedale di Circolo di Busto Arsizio, Varese, Medicina I); Achille Venco, Luigina Guasti, Lorenzo Maroni, Luana Castiglioni (Università degli Studi dell'Insubria, Ospedale di Circolo e Fond Macchi Varese, Medicina Interna I); Massimo Vanoli, Giulia Grignani, Gianluca Casella (Azienda Ospedaliera della Provincia di Lecco Ospedale di Merate, Lecco, Medicina Interna); Gerardo Mancuso, Rosa Tavella, Rosaria Persico (Ospedale Giovanni Paolo II Lamezia Terme (CZ), Unità Operativa Complessa Medicina Interna); Marco Cicardi, Giulia Sandrone (Ospedale Luigi Sacco, Milano, Medicina II); Michele Cortellaro, Marina Magenta, Francesca Perego; Maria Rachele Meroni (Ospedale Luigi Sacco, Milano, Medicina $3^{\circ}$ ); Renzo Rozzini, Lina Falanga, Alessandro Giordano (Ospedale Poliambulanza, Brescia, Medicina Interna e Geriatria); Giorgio Menardo, Stefania Bottone, Elsa Sferrazzo (Ospedale San Paolo, Savona, Medicina Interna e Gastroenterologia); Rodolfo Tassara, Deborah Melis, Lara Rebella (Ospedale San Paolo, Savona, Medicina I); Claudio Ferri, Rinaldo Striuli, Rosa Scipioni (Ospedale San Salvatore, L'Aquila, Medicina Interna Universitaria); Raffaella Salmi, Piergiorgio Gaudenzi, Filippo Di Todaro, Ingrid Nielsen, Lisa Giusto (Ospedale Sant'Anna, Ferrara, Medicina Alta Rotazione); Andrea Semplicini, Lucia Gottardo (Ospedale SS. Giovanni e Paolo, Venezia, Medicina Interna 1); Giuseppe Delitala, Stefano Carta, Sebastiana Atzori (Ospedale Universitario Policlinico di Sassari, Sassari, Clinica Medica); Enrico Agabiti Rosei, Damiano Rizzoni, Luana Castoldi (Spedali Civili di Brescia, Brescia, Seconda Medicina); Emanuele Altomare, Gaetano Serviddio, Santina Salvatore (Ospedali Riuniti di Foggia, Foggia, Medicina Interna Universitaria II); Giacomo Fera, Maria Loreta Di Luca, Donatella Renna (Ospedale San Giacomo di Monopoli, Bari, Unità Operativa Medicina Interna); Antonio Picardi, Chiara Mazzarelli, Umberto Vespasiani Gentilucci, Antonio De Vincentis, Dritan Hila (Università Campus Bio-Medico, Roma, Medicina Clinica e Patologia); Mauro Bernardi, Silvia Li Bassi, Luca Santi (Azienda Ospedaliera Pol- iclinico Sant'Orsola-Malpighi, Bologna, Semeiotica Medica Bernardi); Cesare Masala, Antonio Mammarella, Valeria Raparelli (Policlinico Umberto I, Roma, Medicina Interna D); Filippo Rossi Fanelli, Massimo Delfino, Antonio Amoroso (Policlinico Umberto I, Roma, Medicina Interna H); Pietro Serra, Vincenzo Fontana, Marco Falcone (Policlinico Umberto I, Roma, Terza Clinica Medica); Francesco Violi, Stefania Basili, Ludovica Perri (Policlinico Umberto I, Roma, Prima clinica Medica); Nicolò Gentiloni Silveri, Guido De Marco, Bianca Giupponi (Policlinico Universitario A. Gemelli, Roma, Unità Operativa Complessa Medicina d'Urgenza e Pronto Soccorso); Raffaele Landolfi, Antonio Grieco, Antonella Gallo (Policlinico Universitario A. Gemelli, Roma, Clinica Medica); Francesco Perticone, Angela Sciacqua, Michele Quero, Chiara Bagnato (Università Magna Grecia Policlinico Mater Domini, Catanzaro, Unità Operativa Malattie Cardiovascolari Geriatriche); Paola Loria, Stefano Ballestri, Maria Angela Becchi, Enrica Bellettini (Università Studi di Modena e Reggio Emilia, OCSAE Baggiovara, Modena, Medicina Interna Indirizzo Metabolico Nutrizionistico); Luigi Bolondi, Leonardo Rasciti, Ilaria Serio, Silvia Gualandi (Policlinico Sant'Orsola-Malpighi, Bologna, Unità Operativa Complessa Medicina Interna); Giuseppe Romanelli, Alessandra Marengoni, Francesca Bonometti (Spedali Civili di Brescia, Brescia, Geriatria); Nicola Carulli, Stefania Rondinella, Iolanda Giannico (Medicina Metabolica, Università di Modena e Reggio Emilia); Franco Dallegri, Luciano Ottonello, Alessandra Quercioli, Alessandra Barreca (Medicina Interna 1, Università di Genova); Andrea Sacco, Antonio Bonelli, Gaetano Dentamaro (Medicina, Ospedale Madonna delle Grazie, Matera); Giuliana Micale (Medicina Generale ad indirizzo Geriatrico, IRCCS Istituto Auxologico Italiano, Milano); Giuseppe Delitalia, Silvia Deidda, Luciana Maria Cuccuru (Clinica Medica, Azienda Mista Ospedaliera Universitaria, Sassari); Giampiero Benetti, Michela Quagliolo, Giuseppe Riccardo Centenaro (Medicina 1, Ospedale di Melegnano, Vizzolo Predabissi, Milano); Antonio Perciccante, Alessia Coralli (Medicina, Ospedale San Giovanni-Decollato-Andisilla, Civita Castellana); Cosimo Morabito, Roberto Fava (Medicina, Ospedale Scillesi d'America, Scilla); Luisa Macchini, Anna Realdi (Clinica Medica 4, Università di Padova); Luigi Cricco, Alessandra Fiorentini, Cristina Tofi (Geriatria, Ospedale di Montefiascone).

\section{References}

1 Marengoni A, Angleman S, Melis R, et al: Aging with multimorbidity: a systematic review of the literature. Ageing Res Rev 2011;10:430439.

2 Marengoni A, Fratiglioni L: Disease clusters in older adults: rationale and need for investigation. J Am Geriatr Soc 2011;59:2395-2396.

3 Vogt W, Nagel D: Cluster analysis in diagnosis. Clin Chem 1992;38:182-198.

4 Nobili A, Licata G, Salerno F, et al, SIMI Investigators: Polypharmacy, length of hospital stay, and in-hospital mortality among elderly patients in internal medicine wards: the REPOSI study. Eur J Clin Pharmacol 2011;67: 507-519.
5 World Health Organization: International Classification of Diseases, Injuries, and Causes of Death. Ninth Revision (ICD-9). Geneva, WHO, 1987.

6 Cornell JE, Pugh JA, Williams JW, Kazis L, et al: Multimorbidity clusters: clustering binary data from multimorbidity clusters: clustering binary data from a large administrative medical database. App Multiv Res 2007;12:163182.
7 Gijsen R, Hoeymans N, Schellevis FG, et al Causes and consequences of comorbidity: a review. J Clin Epidemiol 2001;54:661-674.

8 Marengoni A, Rizzuto D, Wang HX, Winblad B, Fratiglioni L: Patterns of chronic multimorbidity in the elderly population. J Am Geriatr Soc 2009;57:225-230.

9 Schäfer I, von Leitner EC, Schön G, et al: Multimorbidity patterns in the elderly: a new approach of disease clustering identifies complex interrelations between chronic conditions. PLoS One 2010;5:e15941.

10 Kirchberger I, Meisinger C, Heier M, et al: Patterns of multimorbidity in the aged population. Results from the KORA-Age study. PLoS One 2012;7:e30556. 
11 Hidalgo CA, Blumm N, Barabási AL, Christakis NA: A dynamic network approach for the study of human phenotypes. PLoS Comput Biol 2009;5:e1000353.

12 Nakauchi H, Tango T, Umesawa $\mathrm{Y}$, et al: Altered immune responsiveness in patients with glomerulonephritis. Microbiol Immunol 1989;33:1013-1025.
13 Thakur H, Gupta L, Sobti RC, et al: Association of GSTM1T1 genes with COPD and prostate cancer in north Indian population. Mol Biol Rep 2011;38:1733-1739.

14 Marengoni A, Bonometti F, Nobili A, et al, Italian Society of Internal Medicine (SIMI) Investigators: In-hospital death and adverse clinical events in elderly patients according to disease clustering: the REPOSI Study. Rejuvenation Res 2010;13:469-477.

15 Nobili A, Marengoni A, Tettamanti M, et al: Association between clusters of diseases and polypharmacy in hospitalized elderly patients: results from the REPOSI study. Eur J Intern Med 2011;22:597-602.
16 Newcomer SR, Steiner JF, Bayliss EA: Identifying subgroups of complex patients with cluster analysis. Am J Manag Care 2011; 17:e324-e332.

17 http://www.ema.europa.eu/docs/en_GB/ document_library/Report/2012/08/WC500 131045.pdf (pp 14-15). 